Review article

\title{
Utilisation of maternity waiting homes and its associated factors in Ethiopia: Systematic reviews and meta-analysis
}

\author{
Bekalu Getnet Kassa *, Alemu Degu Ayele, Habtamu Gebrehana Belay, \\ Gedefaye Nibret Mihiretie, Mulugeta Dile Worke
}

Department of Midwifery, College of Health Sciences, Debre Tabor University, Debre Tabor, Ethiopia

\section{A R T I C L E I N F O}

\section{Keywords:}

Maternity-waiting home

Pooled prevalence

Systematic review

Meta-analysis

Ethiopia

\begin{abstract}
A B S T R A C T
Background: Maternity waiting homes are residential facilities near or in a qualified medical facility where women classified as "high risk" can wait for their delivery before being transferred to a nearby medical facility. Ethiopia has been implementing maternity waiting homes to reduce maternal and prenatal mortality, though utilisation varies significantly between facilities. Thus, this systematic review and meta-analysis was aimed to estimate the pooled prevalence and determinants of maternity-waiting home utilisation in Ethiopia.

Methods: In this study, variables were searched from different electronic database systems, including PubMed/ Medline, CINHAL, Scopus, Web of sciences, google scholar and Grey literature. Data were extracted guided by a standardised data collection measurement tool. The data were analysed using STATA 16 statistical software, and $\mathrm{I}^{2}$ tests assessed heterogeneity between the studies. A random-effect model was used to forecast the pooled utilisation of maternity waiting homes.

Results: Twelve observational studies were included in this review. The pooled prevalence of utilisation of maternity waiting homes in Ethiopia was 44.91\%. Attended antenatal care visits (OR = 2.87, 95\%CI, 1.75-4.69), history of complication ( $\mathrm{OR}=2.05,95 \% \mathrm{CI}, 1.32-3.16)$, travel time to obstetric care facility $>30 \mathrm{~min}(\mathrm{OR}=2.75$, $95 \% \mathrm{CI}, 1.87-4.06$ ), poverty (OR $=4.04,95 \% \mathrm{CI}, 1.46-11.19)$, experience of maternity waiting home use (OR $=$ $2.90,95 \% \mathrm{CI}, 1.14-7.38$ ) and childbirth in the health facility (OR $=11.86,95 \% \mathrm{CI}, 4.02-35.01$ ) were significantly associated with utilisation of maternity waiting home.

Conclusions: This systematic review and meta-analysis revealed that the utilisation of maternity-waiting homes was low in Ethiopia. Thus, concerned stakeholders must design and implement effective interventions to increase maternity-waiting home utilisation and tackle the determinants that deter its utilisation.
\end{abstract}

\section{Introduction}

The global maternal mortality ratio is unacceptably high, with an estimated $42 \%$ of maternal deaths occurring during labour and delivery. ${ }^{1}$ Every day, approximately 800 women die due to pregnancy or childbirth-related complications worldwide. ${ }^{2}$ In 2015, developing regions were responsible for approximately 302,000 global maternal deaths, with Sub-Saharan Africa alone accounting for roughly 201,000, followed by Southern Asia $(66,000) .^{3}$ According to the EDHS 2016, Ethiopia has a high maternal mortality rate of 412 per 100,000 live births and a $26 \%$ institutional delivery rate. ${ }^{4}$
World Health Organization (WHO) has endorsed maternity-waiting home (MWHs) as one of the components of a comprehensive package to reduce maternal morbidity and mortality, ${ }^{5}$ which is essential to most developing countries, where approximately $80 \%$ of the population lives in rural areas, and have lack of access to maternity services, which, in turn, contributes to a high rate of maternal and perinatal mortality., 6 Establishing MWHs for pregnant mothers as a component of antenatal care (ANC) service has been in place for over 100 years, is one of the tried and tested strategies. ${ }^{8}$ The concept was introduced in the early twentieth century, in the United States and Europe, particularly in remote rural areas where women have limited access to an obstetric

; ANC, Antenatal Care;; AOR, Adjusted Odd Ratio; CI, Confidence Interval; EDHS, Ethiopian Demoghrapic and Health Survey; EmOC, Emergency Obstetric Care; FP, Family Planning; MWH, Maternity Waiting Home; OR, Odd Ratio; SNNPR, South Nation Nationality People Region; WHO, World Health Organization.

* Corresponding author.

E-mail addresses: bekalugetnet947@gmail.com (B.G. Kassa), degualemu53@gmail.com (A.D. Ayele), hgebrehana@yahoo.com (H.G. Belay), gedefayen@gmail. com (G.N. Mihiretie), muliedile@gmail.com (M.D. Worke). 
facility. In the 1960 s, maternity waiting homes were introduced into developing countries. ${ }^{9}$ The MWHs are residential facilities near a qualified medical facility where women classified as "high risk"can wait for their delivery before being transferred to a nearby medical facility shortly before delivery or earlier if complications arise. ${ }^{6}$ It provides skilled delivery, postnatal care, referrals in case of complications, counselling for maternal and newborn care, including nutrition and early initiation of breastfeeding and family planning. ${ }^{5}$ It also contributes to addressing the first and second delays in accessing emergency obstetric care (EmOC). The first is a delay in seeking care, while the second is a delay in receiving obstetric care. As a result, $\mathrm{MWH}$ plays a significant role in intervening in those delays. ${ }^{10}$ Thus, implementing an MWH strategy improves access to skilled birth attendance and emergency obstetric care, reducing maternal and perinatal mortality, particularly for women in rural and remote areas. ${ }^{6,11}$

However, in the late 1980s, faith-based organisations pioneered the construction of the first MWHs in Ethiopia to reduce maternal morbidity and mortality and improve postnatal health. In 1985, the initiative was initiated by Attat Hospital in the Gurage Zone of South Nation and Nationalities People of Region (SNNPR). ${ }^{12}$

Different primary studies were conducted in Ethiopia and estimated the prevalence of maternity-waiting home utilisation. ${ }^{13-24}$ According to these separate studies, the prevalence of maternity-waiting home use among Ethiopian women ranges from $7 \%{ }^{16}$ to $68.8 \%,{ }^{17}$ and there was a significant variation and inconsistency in the prevalence of maternity-waiting home use among women across the country. Similarly, the factors associated with the utilisation of MWHs were different from study to study. ${ }^{13-17,19,21-23,25,26}$

The variation mentioned above in the prevalence and determinants of MWH use among Ethiopian women needs to be pooled and utilised at the national level. As a result, it is critical to have summarised evidence on the prevalence rate and determinants of MWH use among women. This assists the relevant bodies in identifying existing gaps and proposing additional strategies to increase the availability, accessibility, and utilisation of MWHs in Ethiopia. Thus, the goal of this study was to summarise the evidence of MWHs utilisation and determinant factors in Ethiopia.

\section{Methods}

\subsection{Data source and searching strategy}

A literature search was performed using Google Scholar, PubMed/ Medline, CINHAL, Scopus, Web of sciences and Grey literature. All studies that reported on maternity-waiting home utilisation and its associated factors in Ethiopia were included. We also performed hand searched for cross-references to distinguish additional relevant articles. The articles were screened using the Preferred Reporting Items for Systematic Review and Meta-Analyses (PRISMA) ${ }^{27}$ (Fig. 1). The search terms and phrases were "Prevalence", "magnitude", "utilisation", "use", "maternity waiting home", "determinants", "predictors", "associated factors", "women", and "Ethiopia"). The search strategies were developed using different logical operators (Additional file 1). Furthermore, to find relevant unpublished studies, Ethiopian universities' digital libraries were searched.

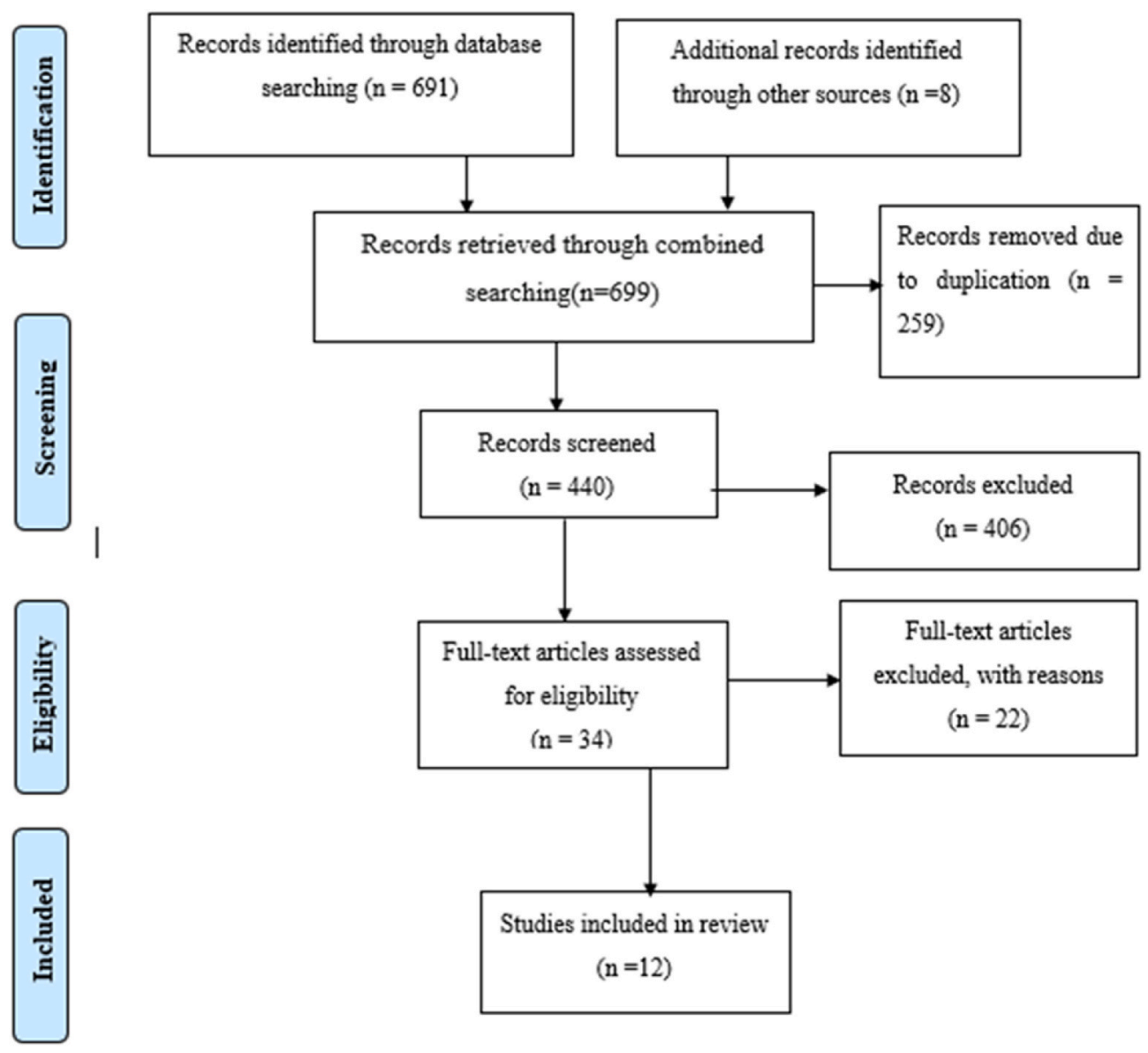

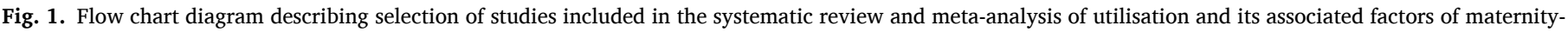
waiting homes among women in Ethiopia. 


\subsection{Criteria for the consideration of studies for the review}

\subsubsection{Inclusion criteria}

Publication status: All published and unpublished studies and studies found on websites of Ethiopian universities and research institutes were included.

Design: All observational studies that measured the utilisation and associated maternity waiting home factors in Ethiopia were included.

Language: Only articles written in the English language were considered.

Publication year: Due to the insufficiency of literature on the utilisation of maternity waiting homes in Ethiopia, we reviewed all research reports until December 30, 2020; we do not have a limitation on starting time of paper publication.

Primary studies scored $\geq 70 \%$ of the Newcastle Ottawa scale criteria for evaluating the quality by three of the authors were included in the meta-analysis. $^{28}$

\subsection{Exclusion criteria}

Studies were excluded: (1) pure qualitative papers that did not include reproductive data for women of childbearing age to be included in the analysis; (2) if the study was not published in English and we were unable to access a copy translated in the English language; (3) cases studies, as most studies lacked robust quality reproductive data to include in the analysis and to reduce the potential for identifying a patient in the review; (4) secondary works (e.g., review articles, commentaries, editorials, or dissertations/thesis, conference abstracts that had not yet been published).

\subsection{Operational definitions}

Exposures: Factors associated with utilisation of MWHs (socio-demographics such as age, religious, educational status, occupational status, socioeconomic status), obstetric related factors (parity, having ANC, history of complication, birth preparedness, knowledge on the history of complication, place of delivery), health care, and behavioural related factor (distance to the health facility, experience of $\mathrm{MWH}$, transport access in obstetric emergencies, Companion for facility visits, barriers MWH utilisation, favourable attitudes towards women's overall evaluation of MWHs, perceived behavioural control of women, positive subjective norms related to women's perceptions of social pressure).

Outcome: MWHs are residential areas near a recognised medical facility where pregnant women with no labour can await the delivery of their baby and be transferred to a nearby medical facility immediately when labour initiates or if any complication arises. The utilisation of MWHs were the primary outcome explored in this systematic review and meta-analysis. It was defined as any woman who used an $\mathrm{MWH}$ regardless of the duration of stay.

\subsection{Data extraction and quality assessment}

All necessary data were extracted from 12 primary studies by three authors (BG, MD and AD) individually using a standardised data extraction form. This form includes primary author, year of publication, study setting, sample size, study design, response rate and prevalence of MWHs are considered. For the second objectives (factors), the information extraction format was prepared for each specific determinant, $i$. e., attended antenatal care visits, history of complication, travel time to obstetric care facility $>30$-min, poverty, experience of maternitywaiting home, wealth status, overall women evaluation of MWHs, perceived behavioural control of women and give childbirth in the health facility. In this study, variables were selected if two or more studies reported them as a significant factor. It was resolved through consensus and by a fourth and fifth investigator during discrepancy in data abstraction between the investigators.
Each incorporated original observational study's scientific strength and quality were assessed using the Newcastle-Ottawa Scale quality assessment tool adapted for cross-sectional study quality assessments. ${ }^{28}$ The tool has three core components; the principal component of the tool graded from five stars and mainly emphasised the methodological quality of each primary study. The second component of the tool graded from two stars mainly concerns each study's comparability, and the last component of the tool graded from three stars and used to assess each original study's outcomes and statistical analysis. The quality of each original study was assessed by three authors independently using this tool. The last two authors resolved disagreements between the three authors. If still there were disagreements between the five authors, the consensus was reached by taking the mean score of the five authors. Finally, the quality of the studies was weighed up based on these components; those with medium (satisfying $70 \%$ of quality evaluation criteria) and high quality ( $\geq 7$ out of 10 scales) were incorporated for analysis $^{29}$ (Additional file 2).

\subsection{Data analysis}

We used Microsoft Excel for data entry and STATA version 16 software for analysis. The associated factors of MWHs were examined based on eligibility criteria. We had considered at least two studies that reported at least one associated factor of MWHs in common with their measure of effect and 95\% confidence interval (CI). The random-effects model based on the DerSimonian-Laird method was considered to assess variations between the studies. The results were presented using texts, tables, and forest plots with effect and 95\% confidence interval measures. Statistical heterogeneity was tested via the $\mathrm{I}^{2}$ statistics at a $p$-value of $\leq 0.05$.

\subsection{Publication bias and heterogeneity}

Rigorous searches (electronic/database search and manual search) have been used to minimise the risk of bias. The authors' cooperative work was also critical in reducing bias, selecting articles based on the clear objectives and eligibility criteria, deciding the studies quality, and extracting and compiling the data. We examine publication bias with a visual inspection of the funnel plot graph qualitatively. Besides, Egger's correlation tests at a 5\% significant level were conducted to assess the presence of publication bias. ${ }^{30,31}$ Furthermore, to reduce the random variations among the primary study's point estimates, subgroup analysis was conducted by study regions, study population and study design. Sensitivity analysis was also performed to identify the potential source of heterogeneity. Heterogeneity across studies was evaluated using inverse variance $\left(\mathrm{I}^{2}\right)$ statistics with its corresponding p-value using the random-effect model.

\section{Results}

\subsection{Description of studies}

We searched around 699 primary studies using electronic medical databases and other relevant sources searching mechanisms. Among the identified studies, 259 articles were removed after reviewing their titles due to duplication, whereas 440 articles were allowed for further screening. Out of these, 404 articles were removed due to irrelevance after being screened based on titles and abstracts. Then after the remaining 34 articles were assessed for eligibility, and 22 of them were excluded due to inappropriate use of statistical analysis, irrelevant target population, inconsistent study report, the outcome of interest not reported, unavailability of full text and inconsistency with the predetermined inclusion criteria for the review. Finally, 12 articles were included in the systematic review and meta-analysis (Fig. 1). 


\subsection{Characteristics of the included studies}

In the current meta-analysis, 9,606 study participants were represented. As described in Table 1, most included studies employed a crosssectional design $(n=10)$, one comparative cross-sectional and one cohort studies study were also included. The prevalence of utilisation of maternity waiting homes reported was between $7 \%{ }^{17}$ and $68.8 \%,{ }^{16}$ and the sample size of the studies ranged from $362^{16}$ to $3,784^{17}$ (Table 1).

\subsection{The utilisation of maternity waiting homes in Ethiopia}

Based on the random effect model, the overall pooled prevalence of utilisation of maternity-waiting homes among women in Ethiopia was 44.91\% (95\% CI: 30.01, 59.82) (Fig. 2).

\subsection{Publication bias and heterogeneity}

Publication biases among the included studies were examined by using both funnel plots and Egger's regression test. The results of funnel plots showed asymmetric shape, which indicates the presence of publication bias among those included studies (Fig. 3A). Objective assessments of publication bias by Egger's regression test also showed the presence of publication bias across studies ( $p$-value $<0.001$ ). The Duval and Tweedie nonparametric trim and fill analysis were done to correct publication bias among the studies. Accordingly, publication bias was corrected when six missed studies were filled in the funnel plot by trim and fill analysis. After six studies were filled, a total of 18 studies were included and computed via the trim and fill analysis to produce the pooled prevalence of $21.429(5.152-37.706)$ by applying a random effect model (Fig. 3B).

\subsection{Sensitivity analysis}

In the current meta-analysis, to determine the potential source of heterogeneity seen in the pooled prevalence of maternity-waiting home utilisation, the investigators performed a leave-one-out sensitivity analysis. The result of the sensitivity analysis indicated that the finding has not relied on a particular study. The pooled prevalence of maternitywaiting home utilisation was varied and ranged from $42.74 \%$ (27.67-57.81\%) to $48.36 \%$ (33.84-62.88\%) after deletion of six studies (Table 2).

\subsection{Subgroup analysis}

In this meta-analysis, we computed subgroup analysis based on the study region. We also executed subgroup analysis using the sample size ( $\leq 500$ and $>500$ ) of the primary studies. The outcome of this subgroup analysis revealed that studies done with a sample size of less than five hundred $56.02 \%$ (95\% CI, 46.14, 65.90) were slightly higher in the utilisation of maternity-waiting home compared to those studies done with a sample size of greater than five hundred, 36.98(17.85, 56.11) (Table 3). Thus, the highest and lowest prevalence was determined in SNNPR and Oromia, which was $40.28 \%$ and $36.16 \%$, respectively (Table 3).

\subsection{Associated factors of the utilisation of maternity waiting home in Ethiopia}

In this review, some of the factors associated with the utilisation of maternity waiting homes were pooled quantitatively, and some were not because of inconsistent classification (grouping) of the exposures concerning the outcome (utilisation maternity waiting home).

Two studies indicated that women who attended antenatal care visits were more likely to utilise maternity waiting homes. Our pooled data indicated that women who have attended antenatal care visits were nearly three times more likely to utilise maternity waiting home than their counterparts (AOR $=2.87,95 \%$ CI: 1.75-4.69) (Fig. 4).

Also, there was an association between history of complication and utilisation of MWH was described in three studies. The pooled analysis demonstrated a statistical difference (AOR $=2.1,95 \%$ CI: $1.03-4.27$ ) (Fig. 4).

Five studies described travel time to obstetric facility $>30 \mathrm{~min}$; one study revealed that there was not associated with utilisation of MWHs; while in four studies, time was found to affect the use of MWHs. The overall pooled estimate indicated that travel time to obstetric facility $>30$ min significantly associated with utilisation of $\mathrm{MWHs}(\mathrm{AOR}=2.71$, 95\%CI: 1.75-4.2) (Fig. 4).

Two studies presented the mother's age with the utilisation of MWHs; all of them was indicated that women in the age group of greater than thirty-one years had an association with utilisation of MWHs. Our pooled estimates showed association with this age ( $\mathrm{AOR}=2.83,95 \% \mathrm{CI}$ : 1.83-4.36) and utilisation of MWHs in Ethiopia (Fig. 4).

Four studies described poverty; all of them were found to affect the use of MWHs significantly. The overall pooled estimate indicated that poverty significantly associated with utilisation of $\mathrm{MWHs}$ (AOR $=3.62$, 95\%CI: 1.16-11.34) (Fig. 4).

Table 1

Summary of characteristics of included studies, 2021.

\begin{tabular}{|c|c|c|c|c|c|c|c|}
\hline Author and year & Study design & Region & Study period & Study population & $\begin{array}{l}\text { Sample } \\
\text { size }\end{array}$ & Response & $\begin{array}{l}\text { Utilisation of } \\
\text { MWH (\%) }\end{array}$ \\
\hline Mekonen et al., 2020 & Cross-section & Amhara & March to May 2018 & Pregnant Women & 525 & 95.05 & 65.3 \\
\hline $\begin{array}{l}\text { Tienke Vermeiden } \\
\text { et al., } 2018\end{array}$ & Cross-section & SNNPR & March and November 2014 & $\begin{array}{l}\text { Recently delivered } \\
\text { women }\end{array}$ & 428 & 100 & 55.1 \\
\hline Kurji J et al., 2019 & Cross-section & Oromia & $\begin{array}{l}\text { September } 1,2015 \text { to } \\
\text { September } 1,2016\end{array}$ & $\begin{array}{l}\text { Recently delivered } \\
\text { women }\end{array}$ & 3784 & 100 & 7 \\
\hline Getachew et al., 2019 & Cross-section & SNNPR & December 2017 to June 2018 & $\begin{array}{l}\text { Recently delivered } \\
\text { women }\end{array}$ & 716 & 100 & 49.7 \\
\hline $\begin{array}{l}\text { Zelalem Tenaw et al., } \\
2019\end{array}$ & Cross-section & SNNPR & April 1-30/2019 & $\begin{array}{l}\text { Recently delivered } \\
\text { women }\end{array}$ & 748 & 99.59 & 67.25 \\
\hline Getnet B et al., 2016 & Cross-section & Oromia & March 06-30/2016 & Pregnant women & 387 & 100 & 38.7 \\
\hline Mengistu A et al., 2019 & Cross-section & $\begin{array}{l}\text { Amhara/Oromia/ } \\
\text { SNNPR/Tigray }\end{array}$ & October-December 2019 & $\begin{array}{l}\text { Recently delivered } \\
\text { women }\end{array}$ & 375 & 100 & 56 \\
\hline Teshale D et al., 2019 & Cross-section & SNNPR & February 20-March 15, 2019 & Pregnant women & 561 & 99.1 & 44.6 \\
\hline $\begin{array}{l}\text { Floris Braata et al., } \\
2017\end{array}$ & Cohort & Oromia/SNNPR & January 2011-December 2014 & Pregnant women & 397 & 100 & 61.5 \\
\hline Kebede et al., 2019 & Cross section & Oromia & March to April 2018 & All women & 362 & 98 & 68.8 \\
\hline $\begin{array}{l}\text { Hailemariam T et al., } \\
2017\end{array}$ & $\begin{array}{l}\text { Comparative cross- } \\
\text { section }\end{array}$ & SNNPR & March 1-15, 2017 & Pregnant women & 516 & 100 & 16.7 \\
\hline Mekides K et al., 2019 & Cross-section & SNNPR & February 1-30, 2019 & All women & 807 & 100 & 8.4 \\
\hline
\end{tabular}




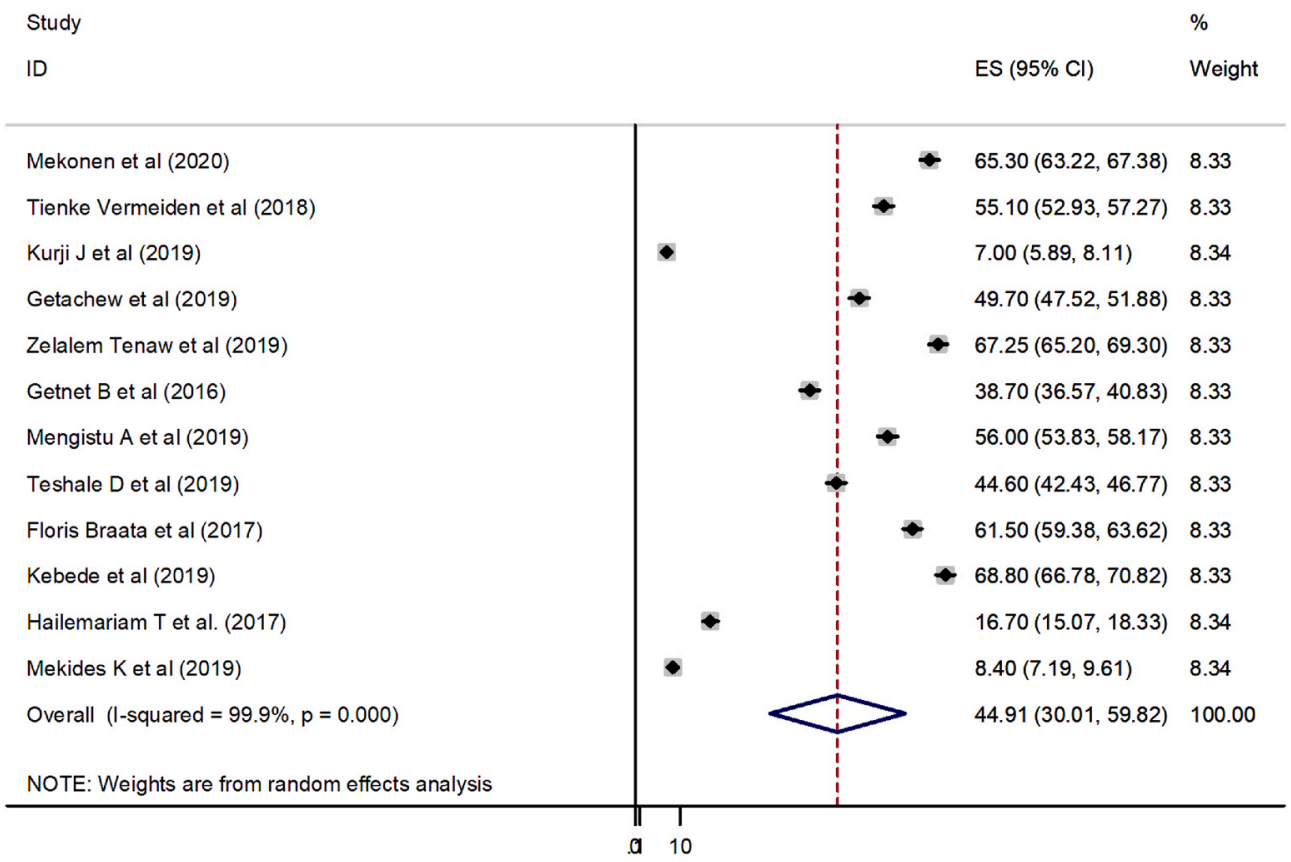

Fig. 2. Forest plot of the pooled prevalence of utilisation of maternity-waiting home among women in Ethiopia, 2021.

Experience of MWHs was assessed in three studies; it was shown that past experienced women were more likely to utilise maternity-waiting homes than those counterparts. The overall estimates revealed that experience of MWHs (AOR $=2.90,95 \%$ CI: 1.14-7.38) was more likely to utilise maternity waiting homes than those counterparts (Fig. 4).

Two studies presented gave childbirth in the health facility with the utilisation of MWHs; both of the studies indicated that women who are giving childbirth in the health facility had an association with utilisation of MWHs. Our pooled estimates showed association with giving childbirth in the health facility (AOR $=11.86,95 \% \mathrm{CI}$ : 4.02-35.01) and utilisation of MWHs (Fig. 4).

\section{Discussion}

The hallmark of utilising a maternity-waiting home includes the prevention of both maternal and neonatal morbidity and mortality. It also has the added benefit of reducing the number of problems, raising healthy generations, and significantly improving the community's overall health. Hence, the current systematic review and meta-analysis aimed to estimate the pooled prevalence of maternity-waiting home utilisation and its determinants among women in Ethiopia.

According to this systematic review and meta-analysis, the pooled prevalence of utilisation of MWHs among women in Ethiopia was $44.91 \%$ (95\% CI: 30.01, 59.82). In this review, the pooled magnitude of the utilisation of MWHs is low. This is in line with individual studies from Tanzania, rural Kenya, and Zambia ${ }^{32-34}$ and higher than the individual studies conducted in Kenya and Zambia. ${ }^{35,36}$ The possible explanations include low socioeconomic status in developing countries, a lack of adequate infrastructure in the health sector, a lack of promotion of MWH use reported in the media, and insufficient attention given to MWH use implementation by the country's health care system. However, it is lower than in individual studies conducted in Kenya's Nyanza Province. $^{37}$ The possible explanation could be differences in socio-cultural parameters, study setting, and time gaps between primary studies conducted in comparable countries. This disparity could be attributed to differences in women's negotiation ability to use MWHs due to socio-cultural impact and respondents' level of knowledge about the unique benefits of using MWHs. Currently, the Ethiopian government is strongly committed to expanding MWH program distributions, which may positively impact utilisation. This implies that an increment of utilisation of maternity homes. Therfore, our government, stakeholders, and mothers should focus and use maternity waiting home to increase service delivery.

This systematic review and meta-analysis also identified the factors associated with the utilisation of MWHs in Ethiopia. Accordingly, women over the age of 31 used MWHs nearly three times more frequently than women under 31 . As far as our knowledge is concerned, this is the new finding. There was no other study that found the same thing or the opposite. This might because that the more that women experience/exposures to health facility services such as ANC and challenges during their previous pregnancy and birth processes and outcomes such as pregnancy complications and delivery, the more users of health services such MWHs. Therefore, attention to the functional health care systems and the community health service systems in orienting the advantage of maternity-waiting homes at the preconception level are needed.

The current study also discovered a significant link between ANC visits and the use of maternity-waiting homes. Compared to their counterparts, women who attend antenatal visits are more likely to use maternity-waiting homes, which is supported by a study conducted in rural Zambia. ${ }^{38}$ Women's use of MWH may have increased due to healthcare workers' advice during ANC visits. It could also be explained that during ANC visits, health care providers clarified the importance of MWHS use.

Another factor influencing maternity-waiting home utilisation is poverty. Poorer women were more represented in the MWH services uses, supported by the study done in Tanzania. ${ }^{32}$ For poorer women, the MWH may be a means to access higher level obstetric care without incurring costs for private transport during labour. This finding also contrary to the study done in Liberia ${ }^{39}$; It is likely that even in rural SSA, the wealthiest women have the economic means for emergency transport to the hospital once labour starts.

Pregnant women at risk of childbirth complications are encouraged to use MWH services because birth complications are unpredictable. ${ }^{40}$ According to the findings of this study, women who had complications during previous births were more likely to use an MWH in the future. This implies that women who have had birth complications may be afraid that they will have similar events during the subsequent 


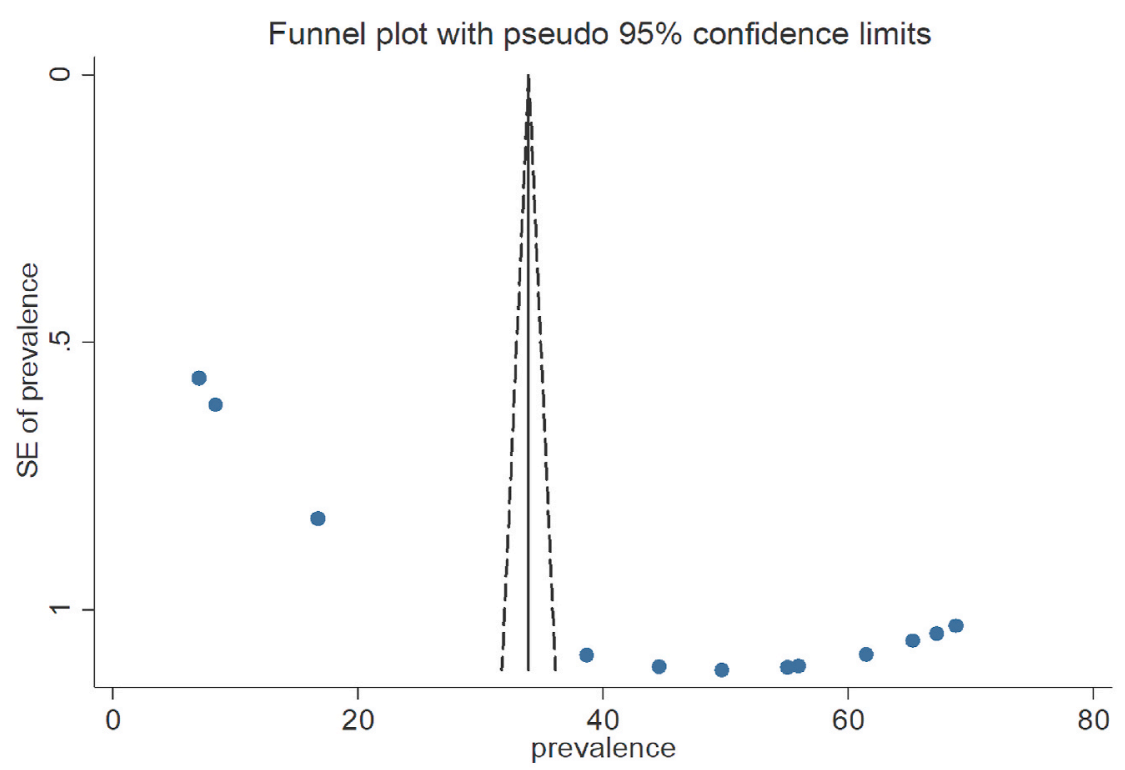

(A)

Filled funnel plot with pseudo $95 \%$ confidence limits

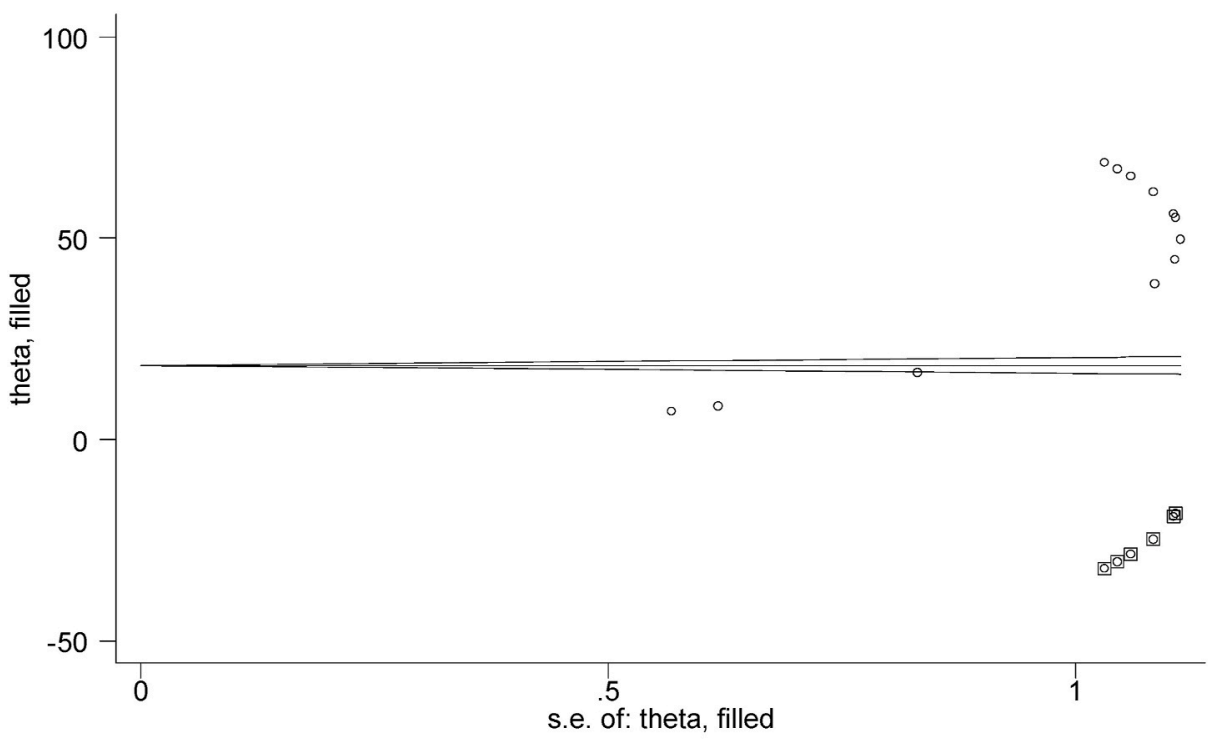

(B)

Fig. 3. (A) Funnel plot to test publication bias of 12 studies. (B) Result of trim and fill analysis for adjusting publication bias of the 18 studies.

Table 2

Sensitivity analysis of the utilisation of maternity-waiting home among women in Ethiopia.

\begin{tabular}{lll}
\hline Study omitted & Pooled Prevalence & 95\% CI \\
\hline Mekonen et al. (2020) & 43.058994 & $27.746393-58.371593$ \\
Tienke Vermeiden et al. (2018) & 43.986752 & $28.222708-59.750793$ \\
Kurji J et al. (2019) & 48.361717 & $33.844997-62.878437$ \\
Getachew et al. (2019) & 44.477661 & $28.563002-60.392323$ \\
Zelalem Tenaw et al. (2019) & 42.8815 & $27.694841-58.068161$ \\
Getnet B et al. (2016) & 45.477566 & $29.365509-61.589622$ \\
Mengistu A et al. (2019) & 43.904922 & $28.170506-59.639336$ \\
Teshale D et al. (2019) & 44.941246 & $28.919382-60.963112$ \\
Floris Braata et al. (2017) & 43.404739 & $27.889658-58.919827$ \\
Kebede et al. (2019) & 42.740372 & $27.667768-57.812973$ \\
Hailemariam T et al. (2017) & 47.478836 & $31.394011-63.563663$ \\
Mekides K et al. (2019) & 48.234509 & $33.082546-63.386475$ \\
\hline
\end{tabular}

childbirth and choose to stay close to health facilities by staying at MWHs.

Although distance remains a potential barrier to healthcare access, MWHs act as a bridge to skilled care by providing temporary shelter near a facility staffed by professionals. ${ }^{41}$ Studies ${ }^{37,41}$ revealed that distance was one factor significantly associated with maternity waiting home. In this study, it is also an associating factor in that women living at a distance that taken a time $30 \mathrm{~min}$ and above from health facility utilised maternity waiting home 2.71 times more likely than women living at a distance that taken time less than $30 \mathrm{~min}$ from health facility which is supported by the study conducted in Zambia ${ }^{34}$ and Tanzania. ${ }^{6,32,42}$ MWH utilisation increased progressively with distance from the health facilities. $^{10}$

Furthermore, MWH experience was a determinant factor in the maternity-waiting home's utilisation. Women who had previous experience with maternity-waiting homes were nearly three times more likely to use MWHs than those who had no prior experience with MWHs. 
Table 3

Subgroup analysis of maternity-waiting home utilisation among women in Ethiopia.

\begin{tabular}{|c|c|c|c|c|c|}
\hline Variables & Characteristics & $\begin{array}{l}\text { Included } \\
\text { studies }\end{array}$ & $\begin{array}{l}\text { Number of } \\
\text { study } \\
\text { participants }\end{array}$ & $\begin{array}{l}\text { Prevalence } \\
(95 \% \text { CI })\end{array}$ & $\begin{array}{l}\mathrm{I}^{2}(\%), \\
P \text {-value }\end{array}$ \\
\hline \multirow[t]{2}{*}{ Region } & Oromia & 3 & 4,533 & $\begin{array}{l}36.16 \\
(-0.09- \\
76.41)\end{array}$ & $\begin{array}{l}99.9, \\
<0.001\end{array}$ \\
\hline & SNNPR & 6 & 3,776 & $\begin{array}{l}40.26 \\
(20.01- \\
60.55)\end{array}$ & $\begin{array}{l}99.9, \\
<0.001\end{array}$ \\
\hline \multirow[t]{2}{*}{$\begin{array}{l}\text { Sample } \\
\text { size }\end{array}$} & $\leq 500$ & 5 & 3,285 & $\begin{array}{l}56.02 \\
(46.14- \\
65.90)\end{array}$ & $\begin{array}{l}99.1, \\
<0.001\end{array}$ \\
\hline & $<500$ & 7 & 5,883 & $\begin{array}{l}36.98 \\
(17.85- \\
56.11)\end{array}$ & $\begin{array}{l}99.9, \\
<0.001\end{array}$ \\
\hline
\end{tabular}

This is due to women's previous experiences/exposures to health facility services such as ANC and challenges during a previous pregnancy and birth processes and outcomes such as pregnancy and delivery complications, which increased their use of health services, including $\mathrm{MWH}$.

Once again, the current meta-analysis findings have revealed that childbirth in a health facility is another factor that influences maternity waiting home utilisation. Previous research has found a link between MWH use and increased facility delivery, antenatal and postnatal attendance. ${ }^{39,41,43}$ This finding is related to our finding that women who had a history of previous health institutional childbirth were more likely to use MWHs than those who gave birth outside the health facility.

Women who have had a previous history of institutional health childbirth (usually due to difficulty during labour) are more likely to receive adequate counselling and advice from skilled birth attendants if they stay at MWHs during their subsequent pregnancies. The women's decisions to use MWHs may have been influenced by lessons learned from previous experiences. In this regard, the role of health professionals in counselling women may be important.

\section{Limitations}

There are some limitations to this systematic review and metaanalysis. Because almost all of the studies included in this systematic review and meta-analysis were cross-sectional, the outcome variables may have been influenced by other confounding variables. Aside from that, some of the studies included in this systematic review and metaanalysis had small sample sizes, which influenced the actual magnitude of utilisation at the country's level. Furthermore, due to a scarcity of studies in Ethiopia, all regions were not represented in this systematic review and meta-analysis (only four regions were represented in this study). As a result, this systematic review and meta-analysis findings may not accurately reflect the national prevalence of maternity-waiting home utilisation.

\section{Conclusions}

In Ethiopia, the overall prevalence of women using maternitywaiting homes is very low. Attendance at antenatal care visits, complication history, travel time to an obstetric care facility $>30$-min, age $>31$ years, poverty, experience with a maternity waiting homes, and childbirth in a health facility were all predictors of maternity-waiting home utilisation. This study suggests that plans and policies to improve childbirth in health facilities and ANC visits at all health system levels should be developed. In addition, it was suggested that the state of the infrastructure be improved.

\section{Authors' contribution}

BG, MD and AD inception and designed the review. BG, HG, and GN developed the search strings and select studies. MD, AD and GN evaluated the quality of the articles. BG, MD and HG performed analysis and interpretation. BG and MD prepared the draft of the manuscript. All authors read and approved the final paper.

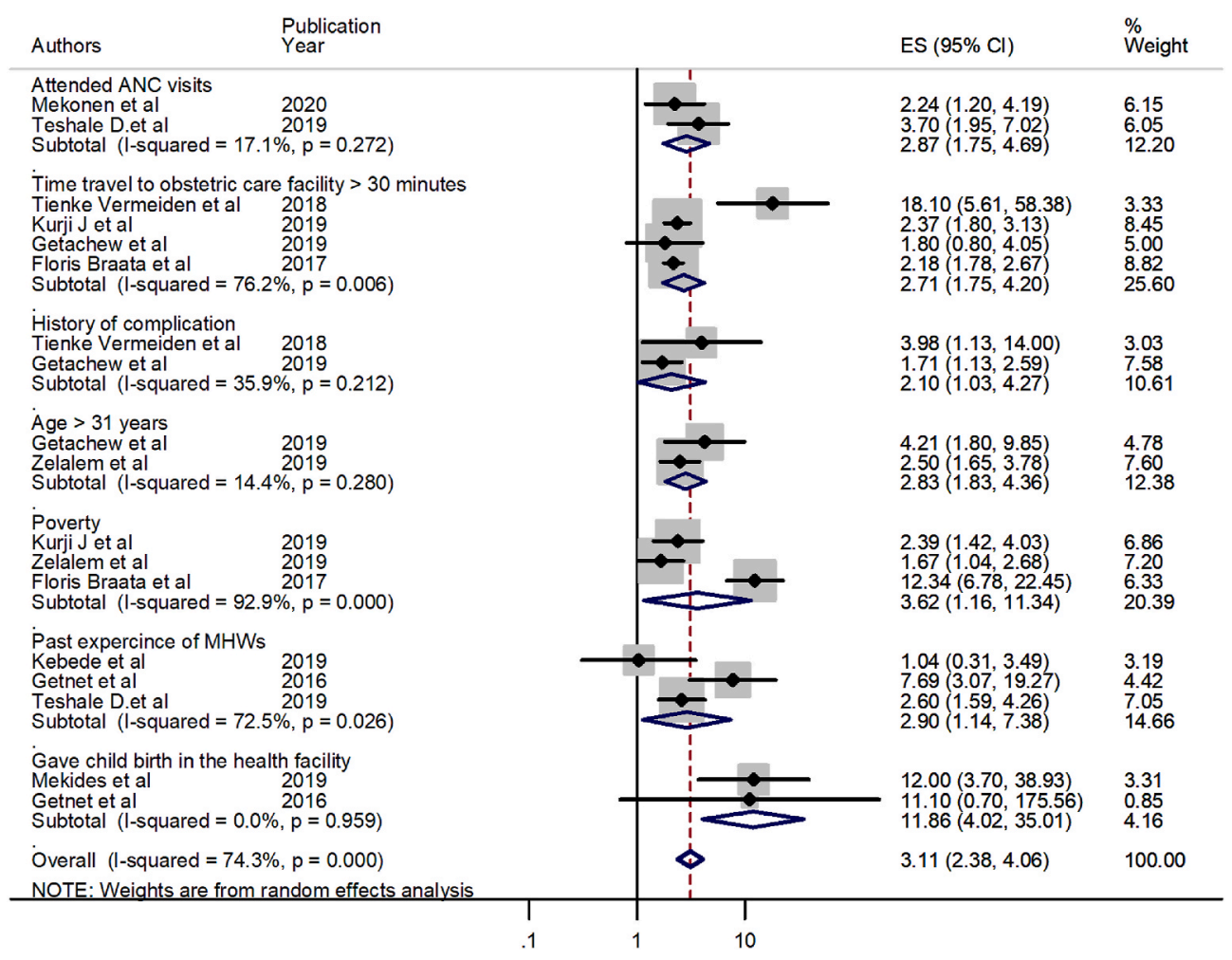

Fig. 4. Factors associated with utilisation of maternity waiting home in Ethiopia. 


\section{Ethics approval and consent to participate}

Not applicable.

\section{Consent for publication}

Not applicable.

\section{Availability of data and materials}

The data that support the review findings of this study are available upon a reasonable request to the corresponding author.

\section{Funding}

Not funded.

\section{Declaration of competing interest}

The authors declare that they have no competing interests.

\section{Acknowledgements}

Not applicable.

\section{Abbreviations}

$\begin{array}{ll}\text { ANC } & \text { Antenatal Care } \\ \text { AOR } & \text { Adjusted Odd Ratio } \\ \text { CI } & \text { Confidence Interval } \\ \text { EDHS } & \text { Ethiopia Demographic and Health Survey } \\ \text { EmOC } & \text { Emergency Obstetric Care } \\ \text { FP } & \text { Family Planning } \\ \text { MWH } & \text { Maternity Waiting Home } \\ \text { OR } & \text { Odd Ratio } \\ \text { SNNPR } & \text { South Nation Nationality People Region } \\ \text { WHO } & \text { World Health Organization }\end{array}$

\section{Appendix A. Supplementary data}

Supplementary data to this article can be found online at https://doi. org/10.1016/j.cegh.2021.100891.

\section{References}

1 Lawn JE, et al. Two million intrapartum-related stillbirths and neonatal deaths: where, why, and what can be done? Int J Gynecol Obstet. 2009;107:S5-S19.

2 WHO. Recommendations on Postnatal Care of the Mother and Newborn. World Health Organization; 2014.

3 WHO. Trends in Maternal Mortality: 1990-2015: Estimates from WHO, UNICEF, UNFPA, World Bank Group and the United Nations Population Division. World Health Organization; 2015.

4 Demographic E. Health Survey, Key Indicators. Rockville: CSA, ICF. 2016.

5 Lori JR, et al. Promoting access: the use of maternity waiting homes to achieve safe motherhood. Midwifery. 2013;29(10):1095-1102.

6 WHO. Maternity Waiting Homes: A Review of Experiences. World Health Organization; 1996.

7 Figa'-Talamanca I. Maternal mortality and the problem of accessibility to obstetric care; the strategy of maternity waiting homes. Soc Sci Med. 1996;42(10):1381-1390.

8 Eckermann E, Deodato G. Maternity waiting homes in Southern Lao PDR: the unique 'silk home'. J Obstet Gynaecol Res. 2008;34(5):767-775.

9 Satti, H., M. McLaughlin, and K. Seung, The role of maternity waiting homes as part of a comprehensive maternal mortality reduction strategy in Lesotho. PIH Reports 2013; 1 (1). Partners In health is a 501 (c)(3) nonprofit corporation and a Massachusetts public charity.@ Partners In Health, 2013. This work is licensed under a Creative Commons Attribution-NonCommercial-NoDerivs 3.0 Unported License. Publication of this report was made possible by flagrant from the Greif Packaging Charitable Trust. Right: Women enjoying the sun outside the maternity waiting home at Nohana: p. 3.

10 van Lonkhuijzen L, Stekelenburg J, van Roosmalen J. Maternity waiting facilities for improving maternal and neonatal outcome in low-resource countries. Cochrane Database Syst Rev. 2012;(10).
11 WHO. Essential Elements of Obstetric Care at First Referral Level. World Health Organization; 1991.

12 Gaym A, Pearson L, Soe K. Maternity waiting homes in Ethiopia-three decades experience. Ethiop Med J. 2012;50(3):209-219.

13 Endalew GB, Gebretsadik LA, Gizaw AT. Intention to use maternity waiting home among pregnant women in Jimma District, Southwest Ethiopia. Glob J Med Res. 2017;16(6):1-9.

14 Endayehu M, Yitayal M, Debie A. Intentions to use maternity waiting homes and associated factors in Northwest Ethiopia. BMC Pregnancy Childbirth. 2020;20:1-10.

15 Getachew B, Liabsuetrakul T, Gebrehiwot Y. Association of maternity waiting home utilization with women's perceived geographic barriers and delivery complications in Ethiopia. Int $J$ Health Plann Manag. 2020;35(1):e96-e107.

16 Kebede Y, et al. User's satisfaction with maternity waiting home services in Jimma zone, Oromia, Ethiopia: implications for maternal and neonatal health improvement. J Women's Health Care. 2019;8(464), 2167-0420.1000464.

17 Kurji J, et al. Factors associated with maternity waiting home use among women in Jimma Zone, Ethiopia: a multilevel cross-sectional analysis. BMJ open. 2019;9(8): e028210.

18 Kurji J, et al. Effectiveness of upgraded maternity waiting homes and local leader training in improving institutional births among women in the Jimma zone, Ethiopia: study protocol for a cluster-randomized controlled trial. Trials. 2019;20(1):1-11.

19 Obola TD, et al. Intention to Use Maternity Waiting Home and Determinant Factors Among Pregnant Women in Misrak Badewacho District, Hadiya Zone, Southern Ethiopia: A Cross Sectional Study. 2019.

20 Tenaw Z, et al. Poverty Reduces Maternity Waiting Home Utilization in Sidama Zone, Southern Ethiopia. 2020.

21 Tiruneh GT, et al. Maternity waiting homes in Rural Health Centers of Ethiop: the situation, women's experiences and challenges. Ethiop J Health Dev. 2016;30(1): $19-28$.

22 Vermeiden T, et al. Factors associated with intended use of a maternity waiting home in southern Ethiopia: a community-based cross-sectional study. BMC Pregnancy Childbirth. 2018;18(1):38.

23 Asnake M, et al. Utilization of Maternity Waiting Homes to Increase Uptake of Immediate Postpartum Family Planning in Primary Health Care Facilities in Ethiopia. 2020.

24 Asmare G, Nigatu D, Debela Y. Factors Affecting Male Partners' Involvement in Maternity Waiting Home Utilization in North Achefer District, Northwest Ethiopia: A Cross-Sectional Study. 2020.

25 Fisseha G, et al. Distance from health facility and mothers' perception of quality related to skilled delivery service utilization in northern Ethiopia. Int $J$ Wom Health. 2017;9:749.

26 Bekele BB, Dadi TL, Tesfaye T. The significant association between maternity waiting homes utilization and perinatal mortality in Africa: systematic review and metaanalysis. BMC Res Notes. 2019;12(1):1-6.

27 Moher D, et al. Reprint preferred reporting items for systematic reviews and metaanalyses: the PRISMA statement. Phys Ther. 2009;89(9):873-880.

28 Zeng X, et al. The methodological quality assessment tools for preclinical and clinical studies, systematic review and meta-analysis, and clinical practice guideline: a systematic review. J Evidence-based Med. 2015;8(1):2-10.

29 Munn Z, Moola S, Lisy K, Riitano D, Tufanaru C. Methodological guidance for systematic reviews of observational epidemiological studies reporting prevalence and cumulative incidence data. Int J Evid-based Healthc. 2015;13(3):147-153.

30 Egger M, et al. Bias in meta-analysis detected by a simple, graphical test. Bmj. 1997; 315(7109):629-634.

31 Sterne JA, Egger M. Funnel plots for detecting bias in meta-analysis: guidelines on choice of axis. J Clin Epidemiol. 2001;54(10):1046-1055.

32 Fogliati P, et al. A new use for an old tool: maternity waiting homes to improve equity in rural childbirth care. Results from a cross-sectional hospital and community survey in Tanzania. Health Pol Plann. 2017;32(10):1354-1360.

33 Mramba L, et al. Reasons for low utilization of a maternity waiting home in rural Kenya. Int J Gynecol Obstet. 2010;108(2):152-153.

34 Lori JR, Boyd CJ, Munro-Kramer ML. Characteristics of maternity waiting homes and the women who use them: findings from a baseline cross-sectional household survey among SMGL-supported districts in Zambia. 2018;13(12):e0209815.

35 Rukia Ware Abdulkadir. Knowledge, attitude and practice of mothers towards skilled delivery services in a maternity waiting home in Merti District, Isiolo County. Prime J Soc Sci. 2015;4(8):1140-1154.

36 Sialubanje C, et al. Improving access to skilled facility-based delivery services: women's beliefs on facilitators and barriers to the utilisation of maternity waiting homes in rural Zambia. Reprod Health. 2015;12(1):61.

37 Creanga AA, et al. Pregnant women's intentions and subsequent behaviors regarding maternal and neonatal service utilization: results from a cohort study in Nyanza Province, Kenya, PloS one 2016:11(9):e0162017.

38 Sialubanje C, et al. Personal and environmental factors associated with the utilisation of maternity waiting homes in rural Zambia. BMC Pregnancy Childbirth. 2017;17(1): 136.

39 Lori JR, et al. Maternity waiting homes and traditional midwives in rural Liberia. Int J Gynecol Obstet. 2013;123(2):114-118.

40 Organization WH. WHO Recommendations on Health Promotion Interventions for Maternal and Newborn Health 2015. World Health Organization; 2015.

41 Henry EG, et al. The influence of quality maternity waiting homes on utilization of facilities for delivery in rural Zambia. Reprod Health. 2017;14(1):68.

42 Straneo M, et al. On the way to universal coverage of maternal services in Iringa rural District in Tanzania. Who is yet to be reached? Afr Health Sci. 2016;16(2):420-428.

43 Lori JR, et al. Maternity waiting homes as part of a comprehensive approach to maternal and newborn care: a cross-sectional survey. BMC Pregnancy Childbirth. 2019;19(1):228. 